\title{
Unique Causes and Manifestations of Eating Disorders Within Transgender Populations
}

SULA MALINA

THEY,THEM

Transgender populations are disproportionately impacted by eating disorders and disordered eating behaviors; however, transgender clients lack access to affirming and culturally responsive mental health care and are frequently undiagnosed. In addition, conventional treatment models for eating disorders do not attend to the unique causes and manifestations of eating disorders among transgender people, which include: minority stress and gender trauma; gender dysphoria and lack of access to safe, gender-affirming treatment; safety concerns and the need for passing; cissexism and resulting disempowerment; and pervasive, harmful beauty standards coupled with hyper-scrutiny of trans bodies. This project includes a summary and analysis of the existing literature and data regarding the causes of and current treatment recommendations for eating disorders within transgender populations. It also suggests a socialwork-led shift within eating disorder treatment to center the sociopolitical forces which so often lead to such diagnoses.

Keywords: transgender, eating disorder, culturally responsive treatment, minority stress, gender trauma, access to care, cissexism, anti-oppressive approach

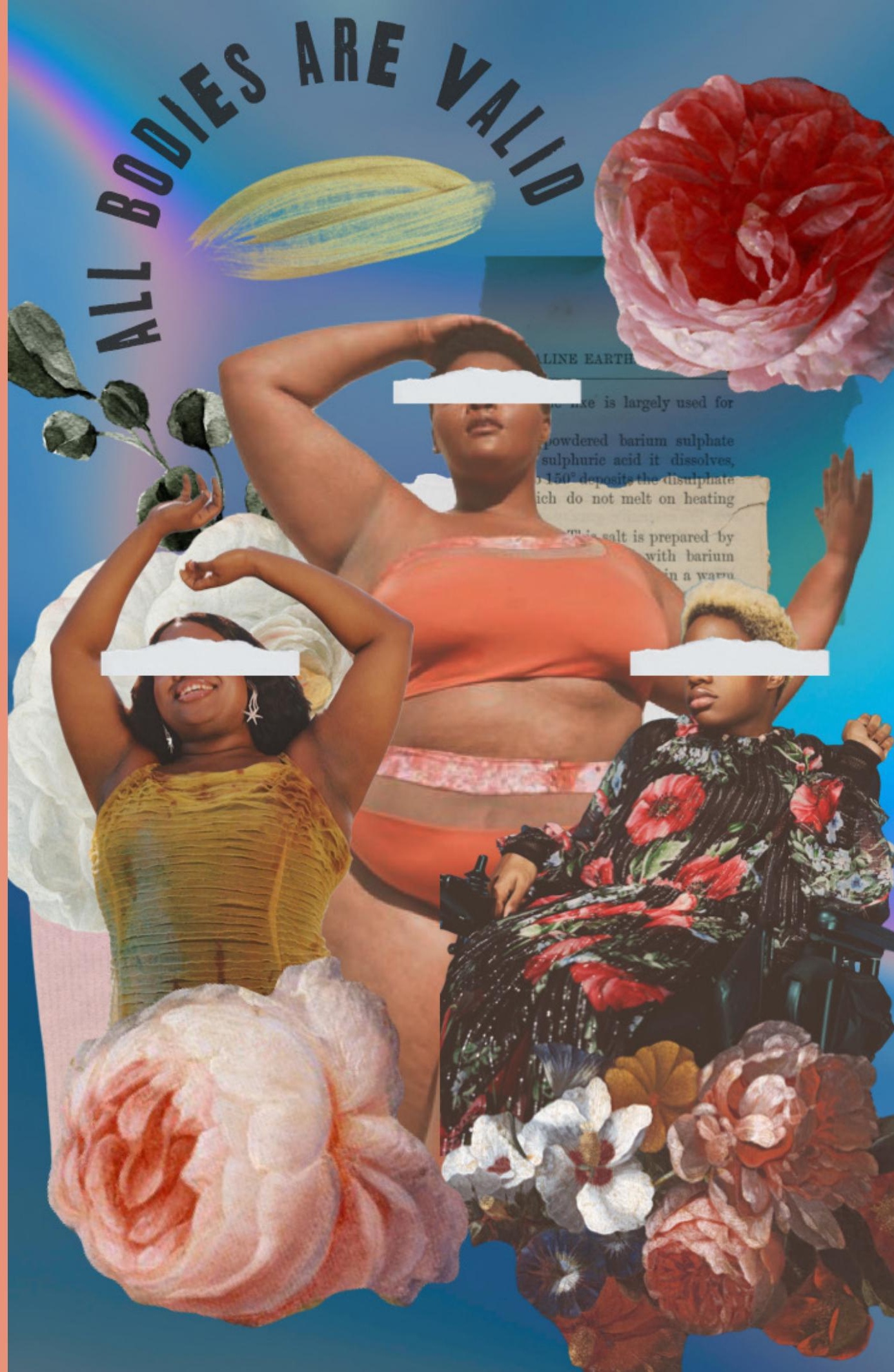




\section{UNIQUE CAUSES AND MANIFESTATIONS OF EATING DISORDERS WITHIN TRANSGENDER POPULATIONS}

Despite limited representation of transgender bodies in both popular media and a lack of attention in clinical training to transgender concerns, research over the past several decades has indicated a high prevalence of eating disorders (EDs) and disordered eating behaviors among transgender populations. Research on health outcomes among transgender people and research on eating disorders are each underfunded (Feldman et al., 2016; Murray et al., 2017); few studies have been published on the intersection of the two that are generalizable at a population level. Those which do exist have focused nearly exclusively on transgender youth. A 2015 study of 289,024 students from 233 U.S. universities revealed that $15.8 \%$ of trans respondents had been diagnosed with an eating disorder, compared to $1.85 \%$ of cisgender, heterosexual women, and $0.55 \%$ of cisgender, heterosexual men (Diemer et al., 2015). The study also collected data on reported disordered eating behaviors among participants within the past month: $13.5 \%$ of trans respondents reported using diet pills within the past month, compared with $4.29 \%$ of cisgender, heterosexual women. Futhermore, $15.1 \%$ of trans respondents reported self-induced vomiting or laxative use within the past month, compared to $3.71 \%$ of cisgender, heterosexual women (Diemer et al., 2015). In collecting symptom-specific data, Diemer et al. identified behaviors in individuals who may not have received a formal diagnosis at the time of data collection due to either their nature or duration. Other Specified Feeding and Eating Disorder (OSFED) is generally considered more common among transgender people than the more widely recognized diagnoses of anorexia nervosa and bulimia nervosa, due to the unique manifestations of disordered eating motivations and behaviors among trans populations. A 2015 study of gender identity, sexual orientation, and self-reported ED diagnoses among college students $(\mathrm{N}=289,024)$ found that trans respondents $(\mathrm{n}=479)$ were more likely to report disordered eating behaviors generally, and particularly those consistent with a diagnosis of OSFED (Diemer et al., 2005). Other researchers have importantly identified that mortality rates for Eating
Disorders Not Otherwise Specified (the DSM-IV diagnosis later renamed OSFED in the DSM 5) are comparable to those for bulimia nervosa (Arcelus, 2011); thus OSFED is no less dangerous than the well-known diagnoses.

Though exact rates vary across research studies, the general trend of higher rates of EDs among trans respondents are consistent. A 2016 study of 218 children and adolescents with gender dysphoria revealed that 13.3\% had "eating difficulties" (Holt et al., 2016), and a 2012 study of 97 youth with "gender identity disorder" (a DSM-IV diagnosis which later became "gender dysphoria" in the DSM 5) demonstrated a 7\% rate of EDs among its sample (Spack et al., 2012). Dangerously little research has been published on the experiences of transgender people of color (POC) navigating eating disorders. Indeed, only $30.42 \%$ of respondents (including only $4.5 \%$ Black and 5.96\% Latinx) to the 2015 study (Diemer et al., 2015) and $11.3 \%$ of respondents to the 2016 study (Holt et al., 2016) were POC. Of the former, only $4.5 \%$ of respondents were Black and $5.96 \%$ were Latinx; the remainder of the $30.42 \%$ were comprised of Asian American Pacific Islander (AAPI), multiracial, Native American, and "unknown" respondents (Diemer et al., 2015). Spack et al. (2012) did not include data around participant race and ethnicity, likely indicating a lack of attention to inclusive participant recruitment, and a fairly racially homogenous sample by extension. In spite of this, high rates of eating disorders among BIPOC populations suggest that rates among trans people of color may be even higher (NEDA, 2018).

These statistics are cause for alarm, not only because of their contrast to data on cisgender youth, but because of the considerable dangers associated with eating disorders. EDs have "the highest rates of related medical complications, hospitalizations, and mortality of all psychiatric disorders" (Duffy et al., 2016, p. 136). This paper seeks to explore the extent to which eating disorders among transgender populations are influenced by sociopolitical forces. Social workers, who are trained to use an anti-oppressive, "social model" of mental health, are uniquely positioned to advocate and provide affirming interventions 
for transgender clients. Clinicians must consider five major contributing factors to eating disorders among transgender populations: 1) minority stress and gender trauma, 2) gender dysphoria and lack of access to safe, gender-affirming treatment, 3) safety concerns and the need for passing, 4) cissexism and resulting disempowerment, and 5) pervasive, harmful beauty standards coupled with hyper-scrutiny of trans bodies.

\section{MAJOR CONTRIBUTING FACTORS}

DISCRIMINATION-BASED STRESS AND GENDER TRAUMA

Minority stress was first introduced in 2003 to describe the result of repeated exposure to microaggressions and other forms of stigma and discrimation among lesbian, gay, and bisexual (LGB) individuals. Epidemiologist Ilan H. Meyer found that high levels of stress were associated with negative mental health outcomes (Meyer, 2003). The concept has since been expanded to other marginalized populations, including Black and Indigenous People of Color (BIPOC), transgender communities, and disabled people, among others. Experts recognize that the experience of transgender embodiment within a cissexist society precipitates gender trauma and stress (Kosciewicz et al., 2020). Moreover, for transgender POC this trauma is compounded by the violence of racism (Harrington, et al., 2006). Researchers have identified a relationship between stress, trauma, and maladaptive coping strategies such as disordered eating (Witcomb et al., 2015, p. 292); high rates of such behaviors and disorders among a population so vulnerable to stress and trauma are, unfortunately, unsurprising.

Despite limited research, there is significant evidence to suggest that the risk of disordered eating among trans people of color is heightened due to the compounding nature of marginalized identities and oppression. Legal scholar Kimberlé Crenshaw introduced the concept of intersectionality in 1991, noting the unique experience of those living at the intersection of multiple marginalized identities, and, consequently, subjugated by multiple systems of oppression. As Crenshaw writes, "the intersectional experience is greater than the sum of racism and sexism" (Crenshaw, 1991, p. 58). Thus, navigating an eating disorder becomes more complex for a transgender person of color than for a white or cisgender person.

\section{GENDER DYSPHORIA AND LACK OF ACCESS TO SAFE, GENDER-AFFIRMING TREATMENT}

Some transgender people experience gender dysphoria: a state of distress caused by the misalignment between their own gender identity and that which is associated with their sex assigned at birth. While the DSM 5 and the World Professional Association of Transgender Health (WPATH) Standards of Care recommend gender-affirming medical intervention such as hormone therapy and surgeries as treatment for gender dysphoria, lack of access to affirming care as well as limited effects of interventions may lead trans individuals to physically "transition" through disordered eating behaviors. For many transgender people, disordered eating can be seen as a method of "either suppressing or accentuating gender by changing the shapes of their bodies" (Kosciewicz et al., 2020, p. 73). For those assigned female at birth, this may mean weight loss to reduce hips, breasts, or buttocks, while those assigned male at birth may gain weight to de-emphasize shoulder breadth, among other characteristics (Kosciewicz et al., 2020). Transmasculine individuals (those assigned female at birth who are transgender and who identify with masculinity to a greater extent than femininity) may restrict their diet to induce amenorrhea, or the cessation of menses (Testa et al., 2017). As Chang et al. (2018) acknowledges, these behaviors, while dangerous, "may feel more accessible or actionable" than physical transition by medical means (p. 116).

Barriers to accessing gender-affirming care may fuel the desire to participate in harmful disordered eating behaviors. Financial limitations may include lack of health insurance coverage, high out-of-pocket cost of care, and limited free time in which to seek care. Geographic restrictions may also create challenges to accessing a gender-affirming provider in close proximity. Finally, lack of support in familial/peer relationships and potential safety risks in altering one's presentation and medical barriers, such as pre-existing conditions that might interfere 
with physical transition or require a particular medical specialist, restrict many transgender people from accessing affirming care.

Physical transformations by way of disordered eating behaviors may be heightened among transgender POC who experience significantly more limited access to gender-affirming medical care, beyond that of their white counterparts (Howard et al., 2019). An analysis of the impacts of such barriers is explored in greater depth in the "Critique of Current Treatment Model" portion of this project.

\section{SAFETY CONCERNS AND THE NEED FOR PASSING}

The concept of passing was initially devised in reference to lightskinned Black Americans who navigated anti-Black racism in the country by presenting themselves as white; historians trace this strategy back to the early years of slavery in the United States (Hobbs, 2014). Passing has since been adopted by transgender communities to refer to the phenomenon by which transgender people are seen by others as cisgender people of their affirmed gender identity. Passing has been rejected by many transgender activists, as to some, the term suggests something inherently "correct" or "successful" about appearing cisgender. Additionally, passing is not achievable for many people, depending on limitations of hormonal and surgical transition as well as gender identity (one might consider what it means to "pass" as nonbinary). It should be noted that passing is not a goal for all transgender people, just as it has certainly not been a goal for all Black people. Many individuals, whether marginalized by transgender identity, race, or both, equate passing to a loss of personal identity and of community/familial ties (Hobbs, 2014).

Although gender dysphoria is understood by behavioral health providers primarily as a mental health concern, passing as one's gender identity has significant societal implications related not only to acceptance, but also to safety. Those "visible" as transgender are particularly susceptible to transphobic discrimination, including social othering, microaggressions, and verbal and physical harrassment and violence. Transgender individuals are socialized to remain hyper- aware of their appearance to onlookers as a matter of survival, and many recognize that "biological sex characteristics related to weight and shape ... may reduce how often they are perceived and treated as the gender they experience themselves to be" (Testa et al., 2017, p. 928). However, passing may precipitate greater safety risks for trans individuals. As activists and theorists alike note, passing as cisgender may be perceived as "deception" by cisgender people (Billard, 2019, p. 463). All too often, "deceived" cisgender people respond to the disclosure of another's transgender status with rage and sometimes fatal violence. Passing as cisgender may be of even greater concern to Black and Brown transgender women, who face an epidemic of violence. In 2020 alone, at least 44 transgender and gender nonconforming people, almost exclusively Black and/or Latinx and transfeminine, were victims of fatal transphobic violence nationally (HRC, 2020). Since 2015, the Human Rights Campaign has recorded a total of 158 deaths (HRC, 2020; HRG, 2019; HRG, 2018; HRG \& TPOCG, 2017; HRC \& TPOCG, 2016; HRC \& TPOCG, 2015). This devastating pattern underscores the complexity of the drive to "pass" (or not) for transfeminine people of color in particular.

The role of passing in driving disordered eating behaviors is complex. One 2018 study of transgender adults $(n=452)$ found a slightly elevated rate of disordered eating among non-binary respondents who had been assigned female at birth, compared to trans men, trans women, and non-binary people assigned male at birth (Diemer et al., 2018). While researchers could not identify a clear cause for the difference, they noted the impact of visible gender-nonconformity (in other words, "lack of passing") often expressed by non-binary trans people and the possibility that some may turn to disordered eating behaviors as a response to the resulting minority stress in a highly binary and conformist society (Diemer et al., 2018). In this sense, EDs may be employed by trans people either as a strategy to control the body's shape and "pass" as a cisgender man or woman, or result from a manifestation of stress and anxiety experienced by those who navigate the world as "unpassable" by virtue of their non-binary gender expression. For some, both factors may be at play. 


\section{CISSEXISM AND RELATED DISEMPOWERMENT}

In response to both pervasive cissexism and gender dysphoria, trans people may turn to disordered eating behaviors as a means of reclaiming a sense of power. As Chang et al. (2018) acknowledge, such behaviors may serve to "provide a sense of control or influence over one's body size or shape" (p. 116). The distress caused by a misalignment between internal identity and the gendered meanings attached to bodies in Western cultures should not be underestimated; indeed, disordered eating may "facilitat [e] a level of omnipotent control in the midst of overwhelming and unbearable somatic feelings, and distress because of one's inability to resolve the conflict between the reality of their gender experience and their heavily defended-against attachment that the body spells as gender's reality" (Kosciewicz et al., 2020, p. 68). Thus, the sense of control some may achieve through disordered eating behaviors may extend beyond those behaviors' visible impact on the shape or size of the body.

\section{HYPER-SCRUTINY OF TRANS BODIES AND PERVASIVE BEAUTY STANDARDS}

Transgender and cisgender people alike risk profound influence by narrow societal beauty standards, though this may be compounded for transgender people who are socialized into a gender role different from their affirmed gender and who may internalize multiple, even contradictory body expectations. As Witcomb et al. (2015) argue, "Trans males may internalize the same ideals that natal females do with regard to the ideal aspects of being female, despite desiring to be male" ( $\mathrm{p}$. 291). The drive for thinness may be compounded by trans identity, given a perceived correlation between weight loss and the "suppress[ion of] features of the birth assigned gender and [accentuation of] the features of the identified gender" (Witcomb et al., 2015, p. 292). Hypervisibility and hyper-scrutiny of trans bodies perpetuates ideals that are even more extreme than those imposed upon cisgender people, "because they are expected to 'prove' themselves as being 'man enough,' 'woman enough,' or 'trans enough'" (Chang et al., 2018, p. 116). These dangerous beauty expectations are based in whiteness, and the fatphobic standards that underlie them, with roots in anti-Black racism. Sociologist Sabrina Strings unravels the history of fatphobia in her text Fearing the Fat Body. Though the current dominant culture in the United States and Europe justifies societal discrimination against fat bodies by deeming them necessarily "unhealthy," this was not the case historically. Indeed, fatness historically came to be associated with "savagery" and "racial inferiority" amidst European colonization of Africa (Strings, 2019, p. 4). The impact this history has had on Black Americans more recently is nuanced. A 2014 series of focus groups comprised of Black women students $(n=31)$ at a large university explored various body image concerns and values among participants. While all participants reported being in some way affected by beauty standards based in whiteness, many also reported that they saw "curviness" as "optimal" for Black women, and considered thinness to be "for white people" (Awad et al., 2016, p. 550). Regardless of its manifestation, there is widespread awareness of body image standards. These standards understandably impact transgender people's relationships with and expectations around their body, shape, and size. Pressures around conforming to beauty standards are compounded significantly for trans POG (Johnson, 2019).

\section{CRITIQUE OF CURRENT TREATMENT MODEL}

The development of gender-affirming, culturally responsive interventions for eating disorders among transgender populations is of paramount importance; however, clients seeking healing face numerous obstacles stemming from a dearth of competent providers, comprehensive research, and safe(r) community spaces.

\section{ACCESSING INCLUSIVE TRANSGENDER COMMUNITY}

For many trans people, and especially trans youth, community may seem altogether nonexistent. As Davis et al. (2018) point out, "the absence of trans-peers and a trans-social network can reinforce the maladaptive behavior that many trans-youth utilize to erase or reconstruct their identities" (p. 56). Even when community is available, the persistent stigma around eating disorders often silences communities 
from healing through necessary conversation. This lack of openness may be explained by the reality that even in their own communities, vocal trans people risk not only "emotional vulnerability," but also "reveal[ing] the fragility of their gender presentation" (Kosciewicz et al., 2020, p. 85). As in many marginalized communities, the value of "pride" in oneself and one's body in the face of systemic violence may backfire when other members feel unable to acknowledge and unpack their internalized oppression.

\section{LIMITING NARRATIVES \& UNDERDIAGNOSIS}

Transgender people are unrepresented in the singular, dominant "eating disorder narrative," which narrowly defines those with eating disorders as thin, white, straight, cisgender women. Consequently, disordered eating behaviors in trans people may go unrecognized--or even vehemently denied--altogether. Kosciewicz et al. (2020) quote one interviewee who explains: "I've been told for so many years that I don't have an eating disorder, there's nothing wrong with me, I'm being dramatic" (p. 83). Research indicates that clients of color, particularly Black clients, are significantly less likely to be diagnosed with an eating disorder when displaying the same eating and exercise behaviors and thought patterns as their white counterparts and non-Black counterparts of color (NEDA, 2018). Underdiagnosis may also be attributed to an over-attribution of symptoms to gender dysphoria because "the conversation about bodies may be so focused on gender that important information is missed" (Chang et al., 2018, p. 115). Certainly the relationship between gender dysphoria and weight dysphoria or body dysmorphia is a nuanced one.

\section{RECONCILING CONTRAINDICATED INTERVENTIONS}

This complex comorbidity of gender dysphoria and body dysmorphia must be explored further. On the surface, the most common treatment approaches to each are in fact contraindicated. Chang et al. (2018) articulates the dangerous potential contradiction clearly:
A common message in society as well as in eating disorder treatment and recovery communities is 'Just accept yourself as you are.' Although this may be an ideal or goal to strive toward regarding body size and weight acceptance, this message can be misapplied in a distorted and harmful way to trans people. It can suggest that trans people should just learn to accept and live in accordance with the gender identity associated with their sex assigned at birth. (p. 117)

This failure to affirm and validate gender identity in eating disorder treatment drives potential patients away from seeking care in the first place. Duffy et al. (2016) report on a study of transgender people with a history of eating disorder treatment, sharing that of the 84 participants, "some even expressed wishing they had never gone to treatment at all, despite acknowledging that it was likely life saving" (p. 144). Gendercompetent care and empathy are critical if providers hope to "heal" their patients from what patients may experience as bringing about affirming physical change and a sense of control. Kosciewicz et al. (2020) emphasize that there is "psychic and physical pain involved in relinquishing the ED as the primary means for self-regulation" (p. 69).

\section{MEDICAL TRANSITION \& GATEKEEPING}

Further, acknowledgement and diagnosis of an eating disorder for a trans patient may prevent access to gender-affirming medical treatments that could alleviate the need for "self-transitioning" behavior. Because trans individuals require clearance from a behavioral health provider to access surgeries, the existence of any mental health diagnoses may halt the process--and, while WPATH Standards of Care do currently clarify that "mental health conditions may be present" (if "reasonably well managed"), "health-care providers may believe that a client should resolve eating-disordered behavior before they are appropriate for undergoing GCMIs" (Testa et al., 2017, p. 928). Such pitfalls would likely be ameliorated by adequate training of medical professionals. At the moment, few training programs offer information that is specific to transgender populations (Duffy et al., 2016). Given this lack of education, 
experiences of eating disorders specific to transgender populations are easily ignored. Popular treatment models for eating disorders often target behavioral changes with insufficient attention to their underlying causes, and can thus be harmful for the transgender populations they may seek to serve. In part, limited research on the subject of transgender patients and eating disorders is to blame. Without an understanding of how access to physical transition can impact mental health outcomes and level of body satisfaction for transgender people, clinicians risk conflating client body dissatisfaction rooted in gender dysphoria with a negative selfimage that centers around size and/or weight. Nutritionists, dieticians, and doctors are limited in their ability to apply nutritional needs to transgender clients, due to a lack of guidelines on the calculation of such needs or ideal body weights for clients who are on hormones (Kosciewicz et al., 2020).

\section{INACCESSIBILITY OF CARE}

Despite recent advancements in the clinical treatment of eating disorders among transgender populations, effective, gender-affirming interventions remain largely inaccessible to the most marginalized trans individuals. Transgender people, and particularly transgender POC, are disproportionately impacted by poverty and homelessness, and thus face significant financial barriers in access to care (National LGBT Health Education Center, 2018). Despite recent policy advocacy, many insurance plans still exclude gender-affirming medical treatments from coverage (National LGBT Health Education Center, 2018). Even for those with access, limitations remain as to what changes existing treatments can facilitate. Although testosterone therapy facilitates body fat redistribution, it brings with it a wide variety of other physical changes with which an individual may not identify (such as facial/body hair or a deeper voice). Though Witcomb et al. (2015) identify that "the body parts that were most reported to cause the most dissatisfaction were those associated with body shape" (p. 291), these may be the very adjustments most difficult to attain through current medical interventions, as they may be "relating to skeletal changes at puberty" that are irreversible (Witcomb et al., 2015, p. 288). Further limitations of medical intervention are demonstrated by the psychological and emotional effects of pubertal suppression on transgender pre-teens, as such intervention can leave them "looking younger than their peers," causing distress (National LGBT Health Education Center, 2018, p.3). While such medical advancements may fall beyond the purview of a social worker, clinicians must be informed on what their transgender clients may experience as deterrents to accessing medical interventions.

\section{PROMISING PRACTICES}

For those working with a younger population in a clinical setting, early intervention is critical. The National LGBT Health Education Center recommends that treatment for eating disorders begin prior to adolescence when possible, in order to prevent long term health consequences (National LGBT Health Education Center, 2018). For those working with transgender clients of any age who experience disordered eating, existing literature suggests a few promising practices: unsettling "diagnosis"; querying "acceptance"; holding space for mourning; and utilizing modalities and frameworks which acknowledge the impact of discrimination-based stress, trauma, and attachment disruption on clients. Given the potential contraindication of healing approaches to eating disorders and gender dysphoria, Chang et al. (2018) recommend that practitioners not designate some patients' concerns as either diagnosis, "but rather as both or an interaction of the two," employing "the dialectic of acceptance and change that is integral to mindfulness-based approaches such as ACT and DBT" (p. 117). Koscieweicz et al. (2020) open a critique of the very notion of mental health diagnosis. As they point out:

We can challenge the normative treatment model of asking clients to learn to love their bodies by dismissing body dissatisfaction as a purely cognitive distortion. For all of our clients (especially trans and GNC people of color) the body exists within a social, political, and historical context that has been a place of both power and violence. ( p. 79) 
By rejecting the "medical model" of mental health in favor of a "social model," practitioners can identify the sociopolitical forces culpable for both gender dysphoria and disordered eating. Acknowledgement of this reality requires that clinicians guide clients through the simultaneous processes of mourning and behavioral shift. As Kosciewicz et al. (2020) write, "this tolerance for the uncertainty, the unknowability of the outcome of mourning, is crucial to the treatment of clients who are reliant on disordered eating behaviors to defend against the body/psyche disjuncture" (p. 69). Utilization of the minority stress framework is one component of anti-oppressive practice, which emphasizes the very real implications of socially constructed (but historically enacted) gender identity and cissexism.

\section{LIMITATIONS}

Existing research on eating disorders among transgender populations is significantly lacking, and that which does exist centers almost entirely on the experiences and diagnoses of white transgender youth. In order to begin to understand the impact of interlocking systems of oppression on transgender people of color, disabled transgender people, and those with other compounding marginalized identities, researchers must dedicate energy to the intentional recruitment of diverse respondents. Additionally, current research largely omits experiences of transgender adults, greatly limiting opportunities for eating disorder professionals to develop best practices when working with those beyond adolescence. Though many sociopolitical factors driving EDs are consistent across age groups, transgender adults may be rendered further vulnerable to disordered eating behaviors if these are driven by gender dysphoria and medical transition has already been "completed." Indeed, much is left to learn regarding the treatment of gender dysphoria for those who have seemingly reached the "limits" of what physical transition (hormonal and surgical) can provide.

\section{CONCLUSION}

This review of existing literature reveals that people who are transgender are disproportionately impacted by disordered eating due to forces that extend far beyond the "purely psychological" (Koscieweicz et al., 2020). Social workers, who embrace an anti-oppressive, "social model" of mental health, are uniquely positioned to advocate for and provide affirming, evidence-based interventions (Koscieweicz et al., 2020). Such interventions reject negative body image related to gender dysphoria as "purely cognitive distortions" and ground treatment in the validation of transgender clients' lived experience with forces of oppression (Koscieweicz et al., 2020). In this sense, social workers have the opportunity not only to address the unique needs of individual clients, but also to carry forward the work of activists past and present committed to dismantling cissexism, racism, sexism, and other forces of oppression in society at large.

\section{REFERENCES}

Arcelus, J., Mitchell, A. J., Wales, J., \& Nielsen, S. (2011). Mortality rates in patients with anorexia nervosa and other eating disorders: A meta-analysis of 36 studies. Archives of General Psychiatry, 68(7), 724-731. doi: 10.1001/archgenpsychiatry.2011.74

Awad, G. H., Norwood, C., Taylor, D. S., Martinez, M., McGlain, S., Jones, B., Holman, A., \& Chapman-Hillard, C. (2014). Beauty and body image concerns among African American college women. Journal of Black Psychology, 41(6), 540-564. doi: $10.1177 / 0095798414550864$

Balsam, K. F., Haug, N. A., Rider, G. N., \& Testa, R. J. (2017). Gender confirming medical interventions and eating disorder symptoms among transgender individuals. Health Psychology, 36(10), 927-936.

Billard, T. J. (2019). "Passing" and the politics of deception: Transgender bodies, cisgender aesthetics, and the policing of inconspicuous marginalized identities. In T. Docan-Morgan (Ed.), The Palgrave handbook of deceptive communication (pp. 463477). Palgrave Macmillan.

Chang, S. C., Singh, A. A., \& Dickey, lore m. (2018). Assess mental health needs. In A clinician's guide to gender-affirming care: Working with transgender \& gender nonconforming clients (pp. 109-122). New Harbinger Publications.

Crenshaw, K. (1991). Demarginalizing the intersection of race and sex: A Black feminist critique of anti-discrimination doctrine, feminist theory, and antiracist politics. In K. T. Bartlett \& R. Kennedy (Eds.), Feminist legal theory: Readings in law and gender (pp. 57-80). Taylor \& Francis. 
Davis, C. (2018). Practice with Transgender People. In G. P. Mallon (Ed.), Social Work Practice with Lesbian, Gay, Bisexual, and Transgender People (3rd ed., pp. 42-65). Routledge.

Diemer, E. W., Grant, J. D., Munn-Chernoff, M. A., Patterson, D. A., \& Duncan, A. E. (2005). Gender identity, sexual orientation, and eating-related pathology in a national sample of college students. Journal of Adolescent Health, 57(2), 144-149. doi: 10.1016/j.jadohealth.2015.03.003

Diemer, E. W., White Hughto, J. M., Gordon, A. R., Guss, C., Austin, S. B., \& Reisner S. L. (2018). Beyond the binary: Differences in eating disorder prevalence by gender identity in a transgender sample. Transgender Health, 3(1), 17-23. doi: 10.1089/ trgh.2017.0043

Duffy, M. E., Earnshaw, V. A., \& Henkel, K. E. (2016). Transgender clients' experiences of eating disorder treatment. Journal of LGBT Issues in Counseling, 10(3), 136-149.

Feldman, J., Brown, G. R., Deutsch, M. B., Hembree, W., Meyer, W., Meyer-Bahlburg, H. F. L., Tangpricha, V., T’Sjoen, G., \& Safer, J. D. (2016). Priorities for transgender medical and health care research. Current Opinions in Endocrinology, Diabetes and Obesity, 23(2), 180-187. doi: 10.1097/MED.0000000000000231

Goldbach, J. T. \& USC LGBT Health Equity Initiative. (2018, March 8). Are you sure more than half of LGBTQ youth have an eating disorder? Because science says otherwise. USC Suzanne Dworak-Peck School of Social Work. https://dworakpeck. usc.edu/news/are-you-sure-more-half-of-lgbtq-youth-have-eating-disorder-becausescience-says-otherwise

Harrington, E. F., Crowther, J. H., Payne-Henrickson, H. C., \& Mickelson, K. D. (2006). The relationships among trauma, stress, ethnicity, and binge eating. Cultural Diversity and Ethnic Minority Psychology, 12(2), 212-229. doi: 10.1037/1099-9809.12.2.212

Hobbs, A. (2014). A chosen exile: A history of racial passing in American life. Harvard University Press.

Holt, V., Skagerberg, E., \& Dunsford, M. (2016). Young people with features of gender dysphoria: Demographics and associated difficulties. Clinical Child Psychology and Psychiatry, 21(1), 108-118. doi: 10.1177/1359104514558431

Human Rights Campaign \& Trans People of Color Coalition. (2015). Addressing antitransgender violence: Exploring realities, challenges and solutions for policymakers and community advocates. http://assets2.hrc.org/files/assets/resources/HRCAntiTransgenderViolence-0519.pdf?_ga=2.37010276.515314532.16137501761124591638.1613750176

Human Rights Campaign \& Trans People of Color Coalition. (2016). A matter of life and death: Fatal violence against transgender people in America 2016. https:// assets2.hrc.org/files/assets/resources/A-Matter-of-Life-and-Death-2016.pdf?_ $\mathrm{ga}=2.37010276 .515314532 .1613750176-1124591638.1613750176$
Human Rights Campaign \& Trans People of Color Coalition. (2017). A time to act: Fatal violence against transgender people in America 2017. http:// assets2.hrc.org/files/assets/resources/A_Time_To_Act_2017_REV3.pdf?_ $\mathrm{ga}=2.37010276 .515314532 .1613750176-1124591638.1613750176$

Human Rights Campaign. (2018). A national epidemic: Fatal anti-transgender violence in America in 2018. https://www.hrc.org/resources/a-national-epidemic-fatal-antitransgender-violence-in-america-in-2018

Human Rights Campaign. (2019). A national epidemic: Fatal anti-transgender violence in the United States in 2019. https://www.hrc.org/resources/a-national-epidemic-fatalanti-trans-violence-in-the-united-states-in-2019

Human Rights Campaign. (2020). Fatal violence against the transgender and gender nonconforming community in 2020. https://www.hrc.org/resources/violence-againstthe-trans-and-gender-non-conforming-community-in-2020

Jackson, D. Z. (2020, August 17). The term "minority" has never made sense. Let's cancel it. Grist. https://grist.org/justice/the-term-minority-has-never-made-sense-letscancel-it/

Johnson, M. Z. (2019, August 30). 10 ways the beauty industry tells you being beautiful means being white. https://thebodyisnotanapology.com/magazine/10-ways-thebeauty-industry-tells-you-being-beautiful-means-being-white/

Kosciewicz, J., Pandjiris, A., \& Zarate, A. (2020). Living in this disembodied body: Navigating the treatment of eating disorders in the transgender and gender nonconforming community. In G. J. Jacobson, J. C. Niemira, \& K. J. Violeta (Eds.), Sex, Sexuality, and Trans Identities: Clinical Guidance for Psychotherapists and Counselors (pp. 67-90). Jessica Kingsley Publishers.

Meyer, I. H. (2003). Prejudice, social stress, and mental health in lesbian, gay, and bisexual populations: Conceptual issues and research evidence. Psychological Bulletin, 129(5), 674-97.

Murray, S. B., Pila, E., Griffiths, S., \& Le Grange, D. (2017). When illness severity and research dollars do not align: Are we overlooking eating disorders? World Psychiatry, 16(3), 321. doi: 10.1002/wps.20465

Mustanski, B. S., Garofalo, R., \& Emerson, E. M. (2010). Mental health disorders, psychological distress, and suicidality in a diverse sample of lesbian, gay, bisexual and transgender youths. American Journal of Public Health, 100(12), 2426-2432. doi: 10.2105/AJPH.2009.178319

National Eating Disorders Association. (2018). People of color and eating disorders. https://www.nationaleatingdisorders.org/people-color-and-eating-disorders

National LGBT Health Education Center (2018, February). Addressing eating disorders, body dissatisfaction, and obesity among sexual and gender minority youth. The Fenway Institute. https://www.lgbthealtheducation.org/wp-content/ uploads/2018/04/EatingDisordersBodyImageBrief.pdf 
NEDA. (2018). People of Color and Eating Disorders. https://www. nationaleatingdisorders.org/people-color-and-eating-disorders

Spack, N. P., Edwards-Leeper, L., Feldman, H. A., Leibowitz, S., Mandel, F., Diamond, D. A., \& Vance, S. R. (2012). Children and adolescents with gender identity disorder referred to a pediatric medical center. Pediatrics, 129(3), 418-425. doi: 10.1542/ peds.2011-0907

Strings, S. (2019). Fearing the Black body: The racial origins of fat phobia. NYU Press.

Witcomb, G. L., Bouman, W. P., Brewin, N., Richards, C., Fernandez-Aranda, F. \& Arcelus, J. (2015). Body image dissatisfaction and eating-related psychopathology in trans individuals: A matched control study. European Eating Disorders Review, 23(4), 287-293.
SULA MALINA (they/them) is a Masters of Science in Social Work candidate at Columbia School of Social Work in Advanced Clinical Practice, concentrating in Health, Mental Health, and Disabilities. Sula holds a Bachelor of Arts in Gender \& Sexuality Studies from Bryn Mawr College. They work as a social work intern at The Gender \& Sexuality Therapy Center in New York City. Originally from Cambridge, Massachusetts, Sula lives in Manhattan. 